\title{
Comparison study of ferrofluid and powder iron oxide nanoparticle permeability across the blood-brain barrier
}

This article was published in the following Dove Press journal:

International Journal of Nanomedicine

12 February 2013

Number of times this article has been viewed

\author{
Dan Hoff' \\ Lubna Sheikh² \\ Soumya Bhattacharya ${ }^{2}$ \\ Suprabha Nayar ${ }^{2}$ \\ Thomas J Webster' \\ 'School of Engineering, Brown \\ University, Providence, RI, USA; \\ ${ }^{2}$ Biomaterials Group, Materials \\ Science and Technology Division, \\ CSIR-National Metallurgical \\ Laboratory, Burmamines, \\ Jamshedpur, India
}

Correspondence: Thomas J Webster

Department of Chemical Engineering,

313A Snell Engineering Center, 360

Huntington Avenue, Boston,

MA 02II5, USA

Tel +16173736585

Fax +l 6173732209

Email th.webster@neu.edu

\begin{abstract}
In the present study, the permeability of 11 different iron oxide nanoparticle (IONP) samples (eight fluids and three powders) was determined using an in vitro blood-brain barrier model. Importantly, the results showed that the ferrofluid formulations were statistically more permeable than the IONP powder formulations at the blood-brain barrier, suggesting a role for the presently studied in situ synthesized ferrofluid formulations using poly(vinyl) alcohol, bovine serum albumin, collagen, glutamic acid, graphene, and their combinations as materials which can cross the blood-brain barrier to deliver drugs or have other neurological therapeutic efficacy. Conversely, the results showed the least permeability across the blood-brain barrier for the IONP with collagen formulation, suggesting a role as a magnetic resonance imaging contrast agent but limiting IONP passage across the blood-brain barrier. Further analysis of the data yielded several trends of note, with little correlation between permeability and fluid zeta potential, but a larger correlation between permeability and fluid particle size (with the smaller particle sizes having larger permeability). Such results lay the foundation for simple modification of iron oxide nanoparticle formulations to either promote or inhibit passage across the bloodbrain barrier, and deserve further investigation for a wide range of applications.
\end{abstract}

Keywords: ferrofluids, iron oxide nanoparticles, permeability, blood-brain barrier

\section{Introduction}

Since its discovery by Ogawa et al in 1990, blood oxygen level-dependent functional magnetic resonance imaging has been used as a method for visualizing neural activity based on blood flow. ${ }^{1}$ Because neurons in various regions fire action potential impulses in response to numerous stimuli, blood flow is regulated to replenish the decrease in oxygen in the local vasculature of the neural response, resulting in a signal increase with a lag time of 2-3 seconds followed by a ramping-up period and plateau for as long as the neurons are active. ${ }^{2}$ Over the past 2 decades, applications of blood oxygen level-dependent functional magnetic resonance imaging have grown from functional mapping of the brain to larger clinical applications, such as assisting in the diagnosis of multiple sclerosis, brain tumors, strokes, and Alzheimer's disease. ${ }^{3}$

With such advances and the significant decrease in the cost of magnetic resonance imaging (MRI) for various diagnostic purposes over the past decade, there has been growing interest in the use of novel contrast agents to improve imaging resolution. Superparamagnetic iron oxide nanoparticles (SPIONs) have been examined as a potentially improved contrast agent given their comparably large magnetic moment and subsequent significant darkening on $\mathrm{T}_{2}$-weighted and $\mathrm{T}_{2}{ }^{*}$-weighted images. This darkening allows for increased image contrast and, by extension, improved MRI 
sensitivity and specificity. Furthermore, the use of SPIONs can be extended if functionalized to image or detect cellular receptors presented in various diseases. ${ }^{4,5}$

However, despite their rise in popularity for MRI imaging purposes, there exists a long-term concern about the use of SPIONs (or any iron oxide nanoparticles) in the body. While some nanoparticles and microparticles are cleared from the body by the reticuloendothelial system, there has been speculation that SPIONs are not cleared as efficiently. While SPIONs are normally coated by a sugar or a polymer, long-term accumulation in the body could eventually lead to degradation of such coatings, exposing the internal iron oxide nanoparticles to the surrounding area. Hence, concerns have arisen about the passage of SPIONs across the blood-brain barrier and accumulation in the brain, which may lead to toxicity, reducing brain function in the long term or worse, lead to progressive neurodegeneration. ${ }^{6}$

In the continuing search for an ideal SPION contrast agent which does not pose such risks, and to increase ease of administration, this in vitro study investigated new SPION fluids (based on poly(vinyl) alcohol [PVA], bovine serum albumin, collagen, glutamic acid, and graphene) and tested their permeability using an in vitro blood-brain barrier model. The results identified several ferrofluids with decreased ability to penetrate the blood-brain barrier which should be further studied for safer MRI applications. At the same time, several iron formulations were found to have improved blood-brain barrier penetration, and should be further studied as a mechanism to cross the blood-brain barrier and treat numerous neurological problems effectively.

\section{Materials and methods Materials}

Anhydrous ferric chloride $\left(\mathrm{FeCl}_{3}\right)$ and liquor ammonia $(30 \% \mathrm{v} / \mathrm{v})$ were purchased from Merck KGaA, (Darmstadt, Germany), hydrated ferrous chloride $\left(\mathrm{FeCl}_{2} \cdot 4 \mathrm{H}_{2} \mathrm{O}\right)$ from Loba Chemie (Mumbai, India), 95\% hydrolyzed PVA (mean molecular weight 95,000) and albumin fraction V (bovine serum albumin) from Acros Organics (Fair Lawn, NJ, USA), and collagen type I and D-glutamic acid from Sigma-Aldrich (St Louis, MO, USA). Fine graphite powder was purchased from Loba Chemie, and potassium dichromate, concentrated $\mathrm{H}_{2} \mathrm{SO}_{4}$, sodium nitrate, and hydrazine hydrate were purchased from Merck. All the chemicals were of analytical grade and used without further purification.
The synthesis of ferrofluids was conducted at the CSIRNational Metallurgical Laboratory in Jamshedpur, India, by a patented process entitled "A biomimetic process for the synthesis of aqueous ferrofluids for biomedical applications." " It is important to note that the ferrofluids mentioned in this paper were synthesized in situ and were not iron oxide particles dispersed in an aqueous medium. In short, the process mainly involves incubation of a ferrous/ferric salt solution in $0.003 \mathrm{M}$ phosphate-buffered saline made inhouse using a water-based salt solution containing sodium chloride, sodium phosphate, potassium chloride, and potassium phosphate supplemented with the additives of interest to this study and further oxidation using ammonium hydroxide under highly alkaline conditions, ie, a variant of the usual chemical coprecipitation method. Reduced graphene oxide was prepared as reported in the literature. ${ }^{8}$ Briefly, potassium dichromate $\left(\mathrm{K}_{2} \mathrm{Cr}_{2} \mathrm{O}_{7}\right)$, concentrated $\mathrm{H}_{2} \mathrm{SO}_{4}$, and sodium nitrate $\left(\mathrm{NaNO}_{3}\right)$ was used for oxidation of graphite to graphene oxide. The graphene oxide synthesized was refluxed at $80^{\circ} \mathrm{C}$ in the presence of hydrazine hydrate to form graphene sheets.

Following synthesis, the fluids were centrifuged at $8000 \mathrm{rpm}$ for 30 minutes as a test of their stability. The supernatant was carefully decanted and dialyzed against $0.001 \mathrm{M}$ phosphate-buffered saline to remove all the byproducts. The precipitates, after repeated washing and vacuum drying at $60^{\circ} \mathrm{C}$, are referred to here as iron oxide nanopowders (IONPs). Eight of the 11 samples tested were ferrofluids containing bovine serum albumin, collagen, PVA, graphene, glutamic acid, and four concentrations $(0.5 \%, 1.5 \%, 2.0 \%$, and $2.5 \%, \mathrm{w} / \mathrm{w}$ ) of PVA containing ferrofluid. The remaining three samples in the solid state were IONP containing PVA, bovine serum albumin, collagen, and graphene. All samples were purely water-based, and the solvent was double-distilled water and phosphate-buffered saline.

\section{Material characterization}

Samples were characterized using X-ray diffraction and ultraviolet-visible spectrophotometry following standard techniques. Confocal images of the fluids were taken using an LSM 700 confocal laser scanning microscope (Zeiss, Oberkochen, Germany) with laser lines at $405 \mathrm{~nm}$ and $488 \mathrm{~nm}$ under $10 \times$ magnification in the bright field mode. All the nanomaterial formulations were also characterized by dynamic light scattering (Malvern Zetasizer Nano Z; Malvern Instruments, Malvern, UK) to assess the hydrodynamic diameter and, by zeta potential, to assess the charge 
of each sample. Each sample was run in $1 \mathrm{~mL}$ cuvettes with five repeat runs per sample.

\section{Confirmation of the blood-brain barrier model}

The objective of this section was to develop an in vitro model of the blood-brain barrier. Characterization via fluorescent sugar transport was used to ensure the blood-brain barrier model met literature standards. For the model, b.End3 (murine brain endothelioma) cells (CRL-2299; American Type Culture Collection, Manassas, VA, USA) were used, and cultured in Dulbecco's modified Eagle medium (DMEM), 10\% fetal bovine serum (FBS) (Sigma-Aldrich), and 1\% penicillin-streptomycin (Life Technologies, Carlsbad, CA, USA). The cells were cultured in a sterile incubator at $37^{\circ} \mathrm{C}$ with $5 \% \mathrm{CO}_{2}$ and $95 \%$ air. Prior to culture on blood-brain barrier inserts, cells were grown to confluency in a BD BioCoat ${ }^{\mathrm{TM}}$ poly-D-lysine vented cap flask (BD Biosciences, Franklin Lakes, NJ, USA).

Next, 6.5 mm Transwell ${ }^{\circledR}$-COL collagen-coated $0.4 \mu \mathrm{m}$ pore polytetrafluoroethylene membrane inserts were used in combination with 24-well plates (BD Biosciences) for the blood-brain barrier model. Each insert was seeded with cells grown to confluency as described in the aforementioned cell culture section. A hemocytometer and a light microscope were used to quantify the concentrations of cells. Cells from the previous section grown to confluency were diluted down to $10^{5}$ cells per $\mathrm{mL}$ in $\mathrm{DMEM}+10 \% \mathrm{FBS}+1 \%$ penicillinstreptomycin, and were pipetted onto the inserts. As per the manufacturer's instructions, $100 \mu \mathrm{L}$ was pipetted onto each insert with $600 \mu \mathrm{L}$ of DMEM $+10 \% \mathrm{FBS}+1 \%$ penicillinstreptomycin. The well plates with inserts were incubated at $37^{\circ} \mathrm{C}$ with $5 \% \mathrm{CO}_{2}$ and $95 \%$ air, and the medium was changed daily in the outer well until cell confluency was attained. After reaching confluency, 1:1 DMEM/Ham's F12 supplemented with $1 \%$ penicillin-streptomycin (henceforth referred to as serum-free medium) was used as the replacement medium for the following 96 hours of experimentation.

To characterize the blood-brain barrier model, b.End3 cells were cultured as already described, and the permeability of the inserts was tested in triplicate using $100 \mu \mathrm{L}$ of $10 \mu \mathrm{g} / \mathrm{mL}$ fluorescein isothiocyanate-labeled dextran (FITC-dextran, molecular weight $3 \mathrm{kDa}$; Sigma-Aldrich) in Hank's Balanced Salt Solution (HBSS; Sigma-Aldrich). Immediately prior to adding FITC-dextran, the inserts were transferred to wells containing $600 \mu \mathrm{L}$ of HBSS. Positive controls were created by adding $100 \mu \mathrm{L}$ of FITC-dextran solution to wells containing $600 \mu \mathrm{L}$ of HBSS. Additionally, $700 \mu \mathrm{L}$ of HBSS was added as a negative control to each well. After the addition of FITCdextran, the well plates were incubated for 2 hours at $37^{\circ} \mathrm{C}$ with $5 \% \mathrm{CO}_{2}$ and $95 \%$ air. Following 2 hours of incubation, the inserts were removed from the outer wells and discarded. Five aliquots of $100 \mu \mathrm{L}$ were taken from each well in the 24-well plate and transferred to a black 96-well plate with a clear bottom (BD Falcon ${ }^{\mathrm{TM}}$; BD Biosciences). The 96-well plate was then inserted into a fluorescent plate reader (GloMax ${ }^{\circledR}$-Multi microplate; Promega, Madison, WI, USA) to be excited at $490 / 20 \mathrm{~nm}$ and measured at 528/20 nm. From the fluorescent plate reader, data were converted into relative fluorescence (RF) and permeability values were determined via equation 1 . For the equation, the volume (V) was $600 \mu \mathrm{L}$; the surface area of the insert (SA) was $0.3 \mathrm{~cm}^{2}$; the time (t) was 2 hours; the concentration of fluid in the donor well $\left(\mathrm{C}_{\mathrm{D}}\right)$ was $10 \mu \mathrm{g} / \mathrm{mL}$; and the maximum possible receiver concentration (ie, the positive control, $\mathrm{C}_{\mathrm{R}, \max }$ ) was $164 \mathrm{pg} / \mathrm{mL}$. Measurements of permeability and the concentration of FITC-dextran in the receiver well $\left(\mathrm{C}_{\mathrm{R}}\right)$ were determined by inputting the RF variable: ${ }^{9}$

$$
\text { Permeability }=\frac{\mathrm{V}}{\mathrm{SA} * \mathrm{t}} * \frac{\mathrm{C}_{\mathrm{R}}}{\mathrm{C}_{\mathrm{D}}}=\frac{\mathrm{V}}{\mathrm{SA} * \mathrm{t}} * \mathrm{RF} * \mathrm{C}_{\mathrm{R}, \max }
$$

Statistics were performed using a one-tailed, heteroscedastic Student's one-tailed $t$-test, with $P<0.01$ considered to be statistically significant.

\section{Experimental samples in the blood-brain barrier model}

To assess the permeability of the various nanomaterial samples, the previously described in vitro model of the blood-brain barrier was used. After 96 hours of exposure to serum-free medium, the inserts were transferred into a 24-well plate (BD Falcon) containing $600 \mu \mathrm{L}$ of HBSS in each well. Each stock sample of the aforementioned nanoparticles was obtained, and diluted 1:19 with HBSS to decrease the concentration to therapeutically relevant levels. The inserts were exposed to the nanoparticles for 2 hours to match the previously described permeability experiment. After 2 hours, the inserts were removed, and an aliquot of $500 \mu \mathrm{L}$ was pipetted from each well into a $20 \mathrm{~mL}$ scintillation vial (Corning, Tewksbury, MA, USA).

In order to prepare the samples to determine their iron concentration (also the nanoparticle concentration), aqua regia was used to convert IONPs into ionic iron. For this, to each scintillation vial, $3 \mathrm{~mL}$ of $12 \mathrm{M} \mathrm{HCl}$ was added dropwise followed by the addition of $1 \mathrm{~mL}$ of $70 \%(\mathrm{v} / \mathrm{v})$ nitric acid. 
Each scintillation vial was then allowed to sit for 30 minutes. After the designated time period, a hot plate was used to boil off the acid. Once all the liquid was removed, the vials were transferred off the hot plate to cool. Next, $4 \mathrm{~mL}$ of $2 \%$ nitric acid was added to each salt vial.

Once iron was in its ionic form, inductively-coupled plasma atomic emission spectroscopy (ICP-AES, JY2000 Ultrac; PerkinElmer, Waltham, MA, USA) was used to determine the iron concentration. The contents of each scintillation vial were emptied into individual plastic tubes for the ICPAES robotic sampler. A standard curve was established from 0-500 ppb to allow for accurate measurements within the range of approximately $20 \mathrm{ppb}$ to $10 \mathrm{ppm}$. A quality control (qc-28) was placed between every 11 samples in an effort to detect whether there was drift in measurement values over the length of the experiment. All experiments were conducted in triplicate and repeated at least three times. Furthermore, one-tailed heteroscedastic $t$-tests were run between the unknown samples and negative controls to establish whether a statistically significant amount of nanoparticles was able to permeate the in vitro blood-brain barrier model.

\section{Results and discussion Material characterization}

Eleven different iron oxide samples were synthesized because of our initial success with $2.5 \%$ PVA ferrofluids as an MRI contrast enhancer in the liver and brain. ${ }^{10}$ The stability and magnetization of $2.5 \%$ PVA ferrofluids was remarkable. ${ }^{10}$ Hence, there was an effort made here to correlate various concentrations and surface functionalities to enable permeability across the present in vitro blood-brain barrier. The fluids synthesized were characterized using X-ray diffraction to confirm iron oxide phase formation with characteristic peaks of magnetite (a representative spectrum can be found in Figure 1). Ultraviolet-visible spectrophotometry was also used to detect the presence of the additives in the 220-280nm range where there is no ferrofluid absorbance (data not shown). The confocal micrographs are very ordered, which again demonstrates the control these additives have on material synthesis (Figure 2).

When observing the distribution of nanoparticles by size, three primary peak ranges were apparent $(20-150 \mathrm{~nm}$, 150-3000 nm, and 3000-7500 nm) when quantified by percent volume. Because this method distorted the true concentration of the nanoparticles by heavily weighting larger particles, a correction factor (inverse of the particle diameter cubed) was applied. A scaling factor was then applied to

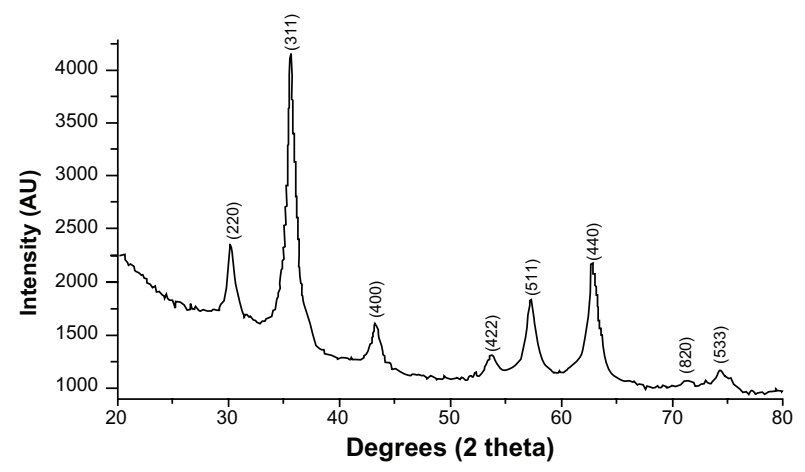

Figure I Representative X-ray diffraction spectra from $2.5 \%$ poly(vinyl) alcohol ferrofluids showing characteristic magnetite peaks.

Note: Similar spectra were observed for all samples, confirming the presence of magnetite.

bring the peaks of each sample to 1.00 for easier observation. After inclusion of these two factors, all peaks in the ppm range decreased to negligible values, while peaks not previously seen in the sub-100 ppb range became visible (Figures 3 and 4). In addition, the average hydrodynamic diameter and zeta potentials of each material of interest to the present study were determined (Figures 5 and 6). Specifically, the results showed the smallest nanoparticles for the ferrofluid formulations. The results further showed the lowest absolute zeta values for IONP with collagen $(-5.36 \mathrm{mV})$, ferrofluids with PVA and glutamic acid $(-3.38 \mathrm{mV})$, and ferrofluids with collagen $(-5.39 \mathrm{mV})$. Further, the highest absolute zeta values were for IONP with graphene and ferrofluids with graphene.

\section{Confirmation of the blood-brain barrier model}

Comparisons of the performance of the experimental bloodbrain barrier model developed here against values in the literature indicated that the model was successfully established (Figure 7). First, the relative fluorescence at 24 hours $\left(\mathrm{RF}_{24}=0.319\right)$ was approximately the same as that described by Bennett et al. ${ }^{11}$ Further, the decline in permeability of about $30 \%$ matched the decline described by Brown et al. ${ }^{12}$ The decline in permeability from increasing exposure to serum-free medium was confirmed as a tightening of the barrier model, which has also been demonstrated by Bennett et al. ${ }^{11}$

\section{Experimental samples in the blood-brain barrier model}

Transport experiments were run with the nanoparticle samples to test whether one could use size, zeta potential, or surface modification as design parameters to moderate permeability. 


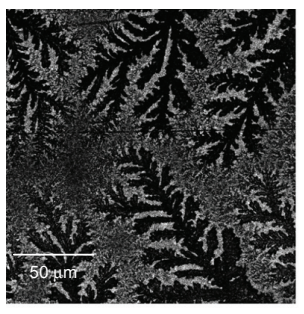

$0.5 \%$ PVA-FF

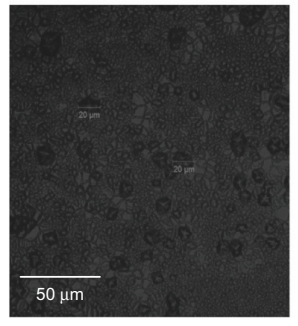

$2.5 \%$ PVA-Glu-FF

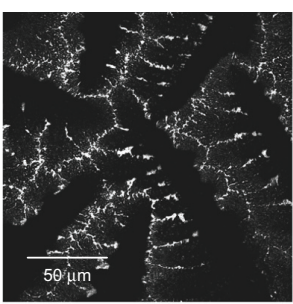

$1.5 \%$ PVA-FF

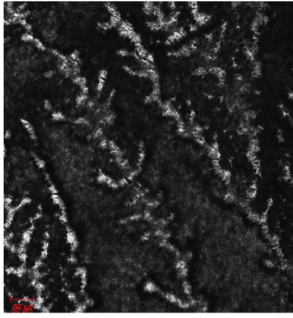

$2.5 \%$ PVA-BSA-FF

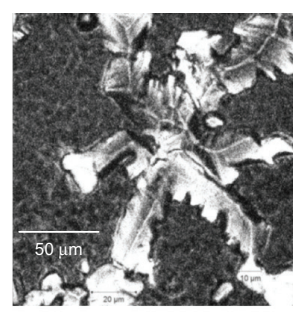

$2.0 \%$ PVA-FF

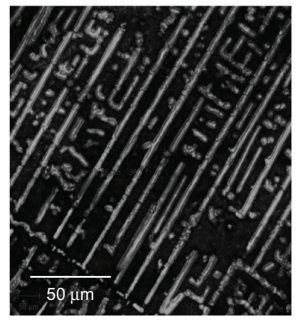

2.5\% PVA-graphene-FF

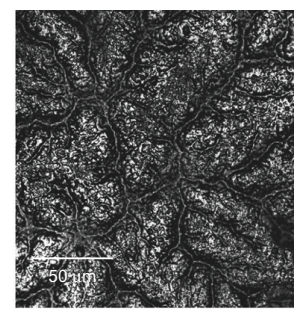

$2.5 \%$ PVA-FF

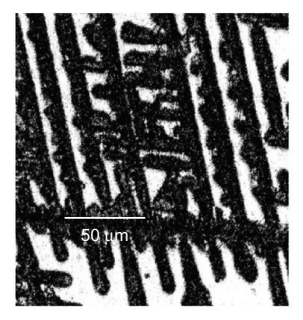

$1.5 \%$ collagen-FF

Figure 2 Representative confocal microscopy pictures for the samples of interest to the present study. Note: Scale bars 50 microns.

Abbreviations: BSA, bovine serum albumin; Glu, glutamic acid; PVA, poly(vinyl) alcohol; FF, ferrofluids.

To control for the presence of background iron, iron passage values were calculated via the following equation:

$$
\text { Passage }_{i}=\frac{\mathrm{C}_{\mathrm{i} \text {,unknown }}-\mathrm{C}_{\mathrm{HBSS}}}{\mathrm{C}_{\mathrm{i}+\text { control }}-\mathrm{C}_{\mathrm{HBSS}}}
$$

where $\mathrm{C}_{\mathrm{i}, \text { unknown }}$ is the average of the experimental wells for a given sample, $\mathrm{C}_{\mathrm{i}+\text { control }}$ is the average of the positive controls for a given sample, and $\mathrm{C}_{\mathrm{HBSS}}$ is the average of the negative controls for the trial. From there, a modified version of the

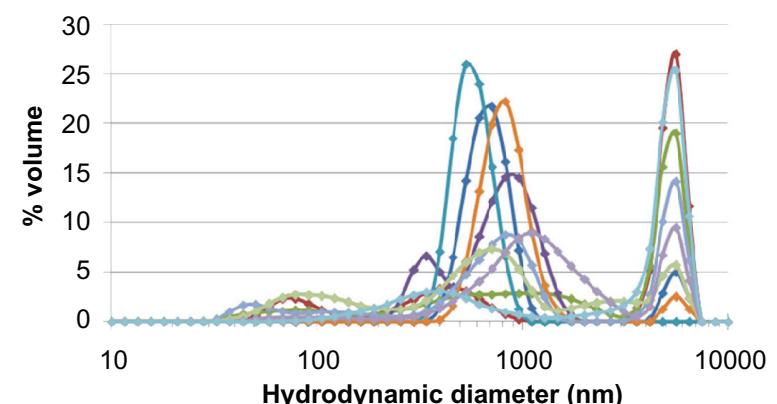

$$
\begin{array}{ll}
\rightarrow-0.5 \% \text { PVA-FF } & \rightarrow-1.5 \% \text { PVA-FF } \\
\rightarrow-2.5 \% \text { PVA-FF } & \rightarrow-2.5 \% \text { PVA-BSA-IONP } \\
\rightarrow-1.5 \% \text { collagen-IONP } & \rightarrow-2.5 \% \text { PVA-graphene-IONP } \\
\rightarrow-2.5 \% \text { PVA-BSA-FF } & \rightarrow-2.5 \% \text { PVA-Glu-FF } \\
\rightarrow-2.0 \% \text { PVA-FF } & \rightarrow-1.5 \% \text { collagen-FF } \\
\rightarrow-2.5 \% \text { graphene-PVA-FF } &
\end{array}
$$

Figure 3 Hydrodynamic diameter by volume.

Abbreviations: BSA, bovine serum albumin; PVA, poly(vinyl) alcohol; FF, ferrofluids; Glu, glutamic acid; IONP, iron oxide nanoparticles. permeability equation used for the FITC-dextran experiment was used to determine permeability. Similar to the FITCdextran permeability equation, the volume $(\mathrm{V})$ was $600 \mu \mathrm{L}$, the surface area of the insert (SA) was $0.3 \mathrm{~cm}^{2}$, and the time (t) was 2 hours: ${ }^{9}$

$$
\text { Permeability }=\frac{\mathrm{V}}{\mathrm{SA} * \mathrm{t}} * \text { Passage } * \mathrm{C}_{+ \text {control }}
$$

The results showed that the lowest permeability was obtained for IONP with collagen; however, at $P<0.01$

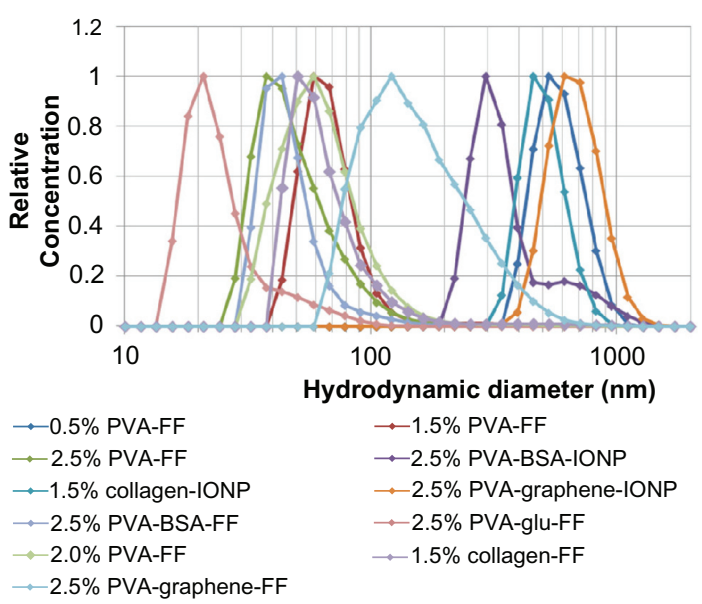

Figure 4 Hydrodynamic diameter by relative concentration.

Abbreviations: BSA, bovine serum albumin; PVA, poly(vinyl) alcohol; FF, ferrofluids; Glu, glutamic acid; IONP, iron oxide nanoparticles. 


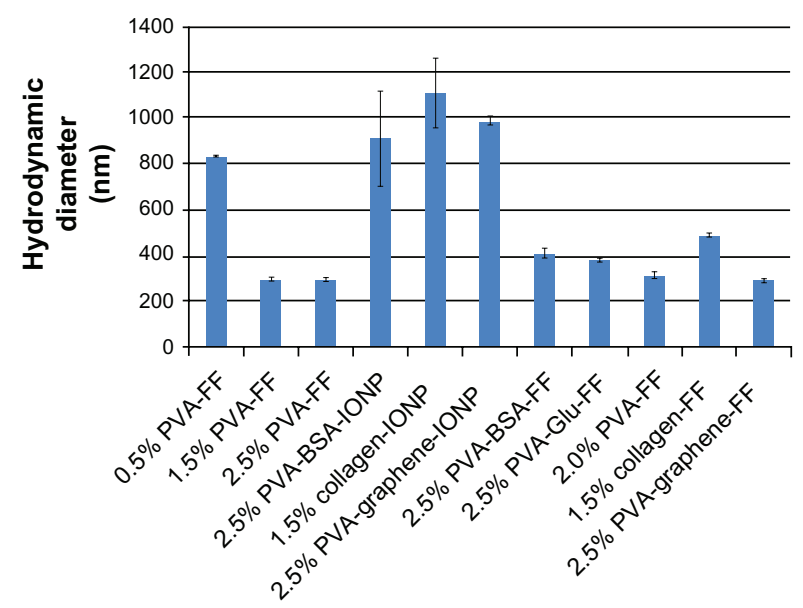

Figure 5 Average hydrodynamic diameters of samples.

Abbreviations: BSA, bovine serum albumin; PVA, poly(vinyl) alcohol; FF, ferrofluids; Glu, glutamic acid; IONP, iron oxide nanoparticles.

(considered to be statistically significant), the permeability of the following samples were not significant from each other: IONP with bovine serum albumin and PVA, IONP with collagen, IONP with graphene and PVA, 0.5\% ferrofluid with PVA, 1.5\% ferrofluid with PVA, 2.0\% ferrofluid with PVA, and $2.5 \%$ ferrofluid with PVA (Figure 8 ). This suggests that IONP should be coated with collagen to avoid penetration of IONP across the blood-brain barrier.

One other statistically significant finding of interest was comparison of the IONP samples with their ferrofluid equivalents (IONP with bovine serum albumin and PVA versus ferrofluid with bovine serum albumin and PVA, IONP with collagen versus ferrofluid with collagen, and IONP with graphene and PVA versus ferrofluid with graphene and PVA). A two-tailed heteroscedastic $t$-test revealed that the

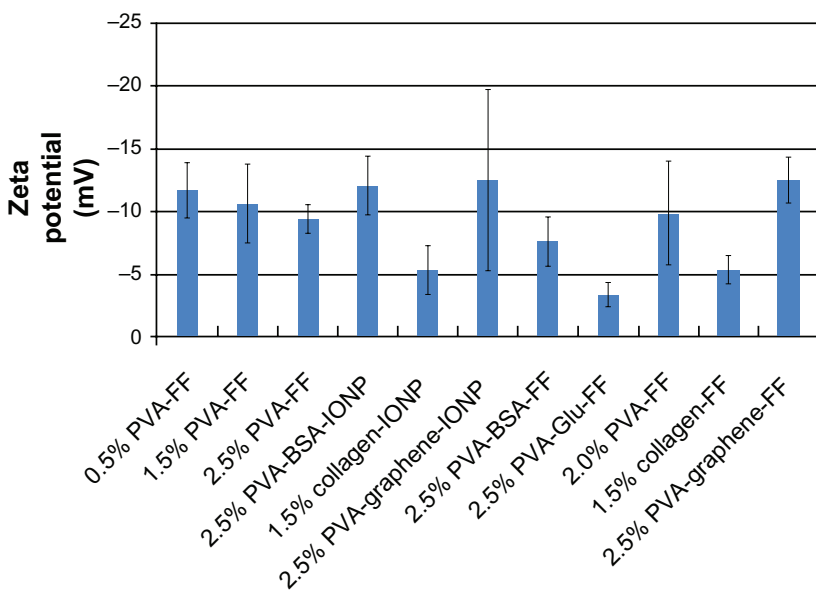

Figure 6 Average zeta potential of samples.

Abbreviations: BSA, bovine serum albumin; PVA, poly(vinyl) alcohol; FF, ferrofluids; Glu, glutamic acid; IONP, iron oxide nanoparticles.

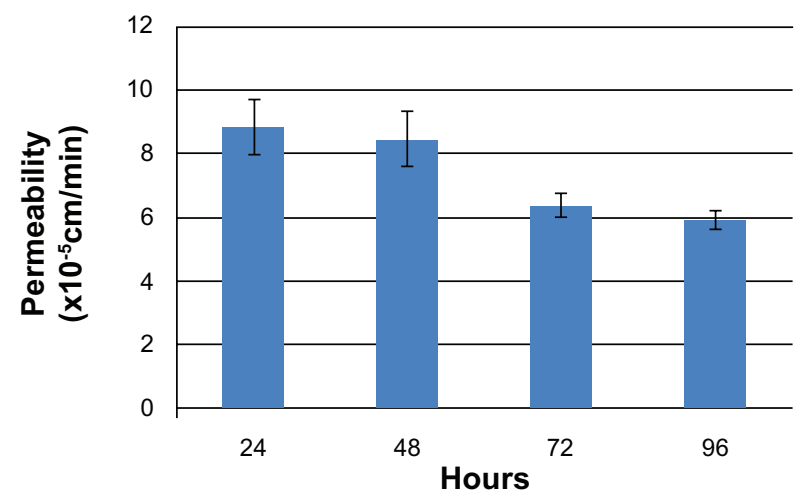

Figure 7 Permeability of fluorescein isothiocyanate-labeled dextran across the b. End3 confluent blood-brain barrier model membrane confirming establishment of an accurate blood-brain barrier.

ferrofluid formulations were significantly more permeable than the IONP formulations, suggesting a role for the presently studied ferrofluid formulations in crossing the bloodbrain barrier to deliver drugs or have additional neurological therapeutic efficacy. Although an argument can be made that the large value of ferrofluid with collagen drives this finding, the significance is supported by a $P$-value of 0.052 upon removing IONP with collagen and ferrofluid with collagen from this analysis.

As a further analysis of why blood-brain barrier permeability changed with respect to IONP size, a regression was run on hydrodynamic diameter and permeability (Figure 9). An exponential trend line yielded the best fit, with an $\mathrm{R}^{2}$ value of 0.286 (equivalent $P$-value 0.090 ). This marginally supports an initial hypothesis that larger particles have increased difficulty negotiating the blood-brain barrier.

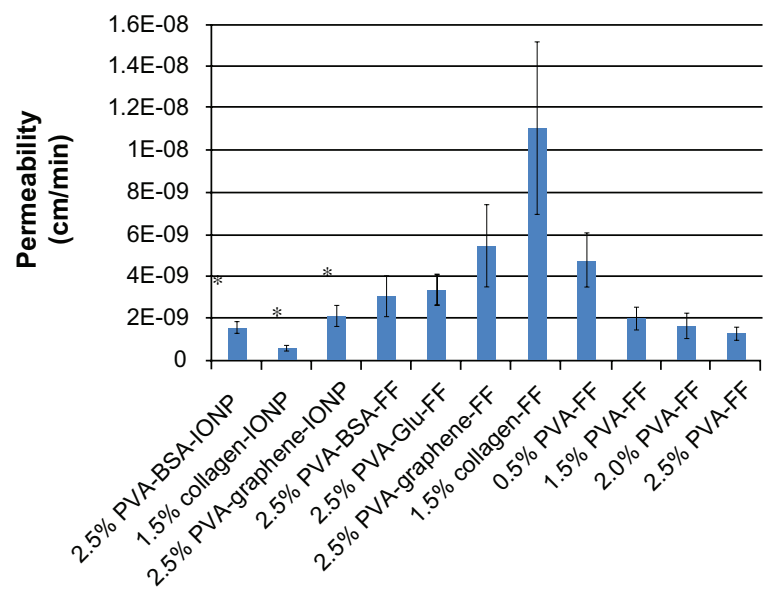

Figure 8 Blood-brain barrier permeability by sample.

Notes: Data are shown as the mean \pm standard error of the mean; $n=3 ; * P<0.01$ compared with respective ferrofluid sample.

Abbreviations: BSA, bovine serum albumin; PVA, poly(vinyl) alcohol; FF, ferrofluids; Glu, glutamic acid; IONP, iron oxide nanoparticles. 


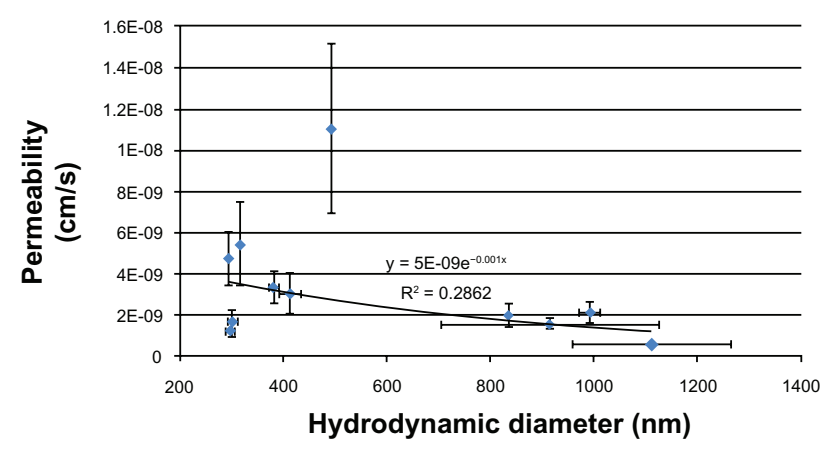

Figure 9 Permeability of nanoparticles by size.

Although the presence of the ferrofluid with collagen and PVA sample appears to be an outlier and a cause of the poor low $\mathrm{R}^{2}$, its removal only increases the fit to an $\mathrm{R}^{2}$ value of 0.315 ( $P$-value equivalent 0.073 ). Of course, the mechanism of diffusion (transcytosis, extracellular, toxic response) cannot be stated for certain with the analytical methods used. Nonetheless, size has been shown to be relevant to the diffusion process across the blood-brain barrier.

The zeta potential was also assessed to determine whether surface energy could be used to mediate permeability across the blood-brain barrier (Figure 10). While linear regression indicated a positive trend, the correlation was rather weak, with an $\mathrm{R}^{2}$ value of 0.089 ( $P$ value equivalent 0.374 ). Although this low correlation may not support the hypothesis that the high energy particles formulated here can permeate the blood-brain barrier, future studies should be conducted, because surface energy, particle size, and chemistry changed simultaneously in this study, making such correlations difficult. Similar to other studies, the present results demonstrate an ability to control material properties which either penetrate or do not penetrate the blood-brain barrier. ${ }^{13-15}$

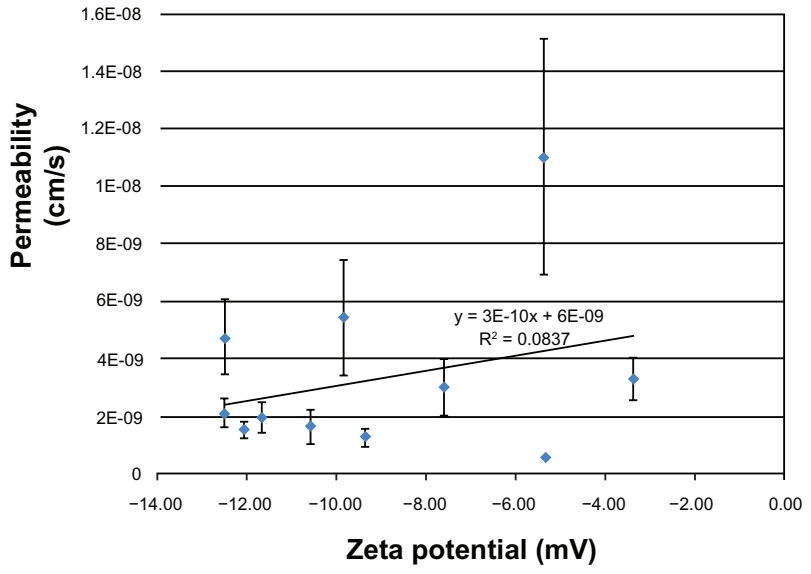

Figure 10 Permeability of nanoparticles by zeta potential.

\section{Conclusion}

In the present study, an in vitro model of the blood-brain barrier based on b.End3 cells was established. Viability of the model was confirmed by comparison of the permeability of FITC-dextran at increasing lengths of time in culture in serum-free medium with values reported in the literature. Having successfully developed a blood-brain barrier model, the permeability of 11 different types of IONPs and fluids was determined across this in vitro blood-brain barrier. Importantly, the results show that the ferrofluid formulations were significantly more able to permeate the blood-brain barrier than the IONP formulations, suggesting a role for the presently studied in situ synthesized ferrofluid formulations (ferrofluid with PVA, ferrofluid with collagen, ferrofluid with bovine serum albumin and PVA, ferrofluid with glutamic acid and PVA, and ferrofluid with graphene and PVA) as a material to cross the blood-brain barrier for the delivery of drugs or have another type of neurological therapeutic efficacy. Conversely, the results showed the least permeability for IONP coated with collagen, suggesting its role as an MRI contrast agent but with limited IONP passage across the blood-brain barrier. Further analysis of the data yielded several trends of note, with little correlation between permeability and zeta potential of the formulation, but a larger correlation between permeability and particle size (with smaller particles having greater permeability). Such results lay the foundation for simple modification of iron oxide formulations to either promote or inhibit passage across the blood-brain barrier, which deserves further investigation.

\section{Acknowledgment}

The authors would like to thank the Hermann Foundation for funding this research.

\section{Disclosure}

The authors report no conflicts of interest in this paper.

\section{References}

1. Ogawa S, Lee T, Kay AR, Tank DW. Brain magnetic resonance imaging with contrast dependent on blood oxygenation. Proc Natl Acad Sci US A. 1990;87:9868-9872.

2. Pisanic TR III, Blackwell JD, Shubayev VI, Finones RR, Jin S. Nanotoxicity of iron oxide nanoparticle internalization in growing neurons. Biomaterials. 2007;28:2572-2581.

3. Babes L, Denizot B, Tanguy G, Le Jeune JJ, Jallet P. Synthesis of iron oxide nanoparticles used as MRI contrast agents: a parametric study. J Colloid Interface Sci. 1999;212:474-482.

4. Berry CC, Curtis ASG. Functionalisation of magnetic nanoparticles for application in biomedicine. J Phys D Appl Phys. 2003;36:198-206.

5. Iannetti GD, Wise RG. BOLD functional MRI in disease and pharmacological studies: room for improvement? Magn Reson Imaging 2007;25:978-988. 
6. Logothetis NK, Pauls J, Augath M, Trinath T, Oeltermann A. Neurophysiological investigation of the basis of the fMRI signal. Nature. 2001;412:150-157.

7. Nayar S, Sinha A, Pramanick AK, inventors. A biomimetic process for the synthesis of aqueous ferrofluids for biomedical applications. Application number 0672DEL2010. March 22, 2010

8. Chandra S, Sumanta S, Panchanan P. A novel synthesis of graphene by dichromate oxidation. Materials Science and Engineering B. 2010; 167:133-136.

9. Manufacturer instructions, BD Biosciences, 2011. Franklin Lakes, NJ, USA. November 1, 2101.

10. Nayar S. Improved MRI contrast enhancer in the brain and liver through the use of ferrofluids. Presented at the MRS Annual Conference, Boston, MA, USA. December 3, 2011.

11. Bennett J, Basivireddy J, Kollar A, Biron KE, McQuaid S. Blood-brain barrier disruption and enhanced vascular permeability in the multiple sclerosis model EAE. J Neuroimmunol. 2010;229:180-191.
12. Brown RC, Morris AP, O’Neil RG. Tight junction protein expression and barrier properties of immortalized mouse brain microvessel endothelial cells. Brain Res. 2007;1130:17-30.

13. Gulati K, Sinn Aw M, Losic D. Nanoengineered drug-releasing Ti wires as an alternative for local delivery of chemotherapeutics in the brain. Int J Nanomedicine. 2012;7:2069-2076.

14. Saiyed ZM, Gandhi NH, Nair MPN. Magnetic nanoformulation of azidothymidine 5 -triphosphate for targeted delivery across the bloodbrain barrier. Int $J$ Nanomedicine. 2010;5:157-166.

15. Dan M, Tseng MT, Wu P, Unrine JM, Grulke EA, Yokel RA. Brain microvascular endothelial cell association and distribution of a $5 \mathrm{~nm}$ ceria engineered nanomaterial. Int J Nanomedicine. 2012;7:4023-4036.
International Journal of Nanomedicine

\section{Publish your work in this journal}

The International Journal of Nanomedicine is an international, peerreviewed journal focusing on the application of nanotechnology in diagnostics, therapeutics, and drug delivery systems throughout the biomedical field. This journal is indexed on PubMed Central, MedLine, CAS, SciSearch ${ }^{\circledR}$, Current Contents ${ }^{\circledR} /$ Clinical Medicine,

\section{Dovepress}

Journal Citation Reports/Science Edition, EMBase, Scopus and the Elsevier Bibliographic databases. The manuscript management system is completely online and includes a very quick and fair peer-review system, which is all easy to use. Visit http://www.dovepress.com/ testimonials.php to read real quotes from published authors. 